\title{
LOGÍSTICA REVERSA: ANÁLISE BIBLIOMÉTRICA DE ARTIGOS PUBLICADOS EM PERIÓDICOS BRASILEIROS NO PERÍODO DE 2003 A $2012^{1}$
}

\author{
REVERSE LOGISTICS: BIBLIOMETRIC ANALYSIS OF BRAZILIAN JOURNAL \\ PUBLISHED IN PERIOD FROM 2003 TO 2012
}

\author{
Ferdinando David Valandro \\ Especialista em Finanças e Controladoria \\ Instituto Nacional de Pós-Graduação (INPG) \\ ferdinando.valandro@brandili.com.br \\ Marcia Zanievicz Silva ${ }^{2}$ \\ Doutora em Ciências Contábeis e Administração pela FURB \\ Professora do Programa de Pós-Graduação em Ciências Contábeis e Administração da FURB \\ marciaza@gmail.com \\ Júlio César Silva \\ Doutorando em Ciências Contábeis e Administração \\ Fundação Universidade Regional de Blumenau (FURB) \\ profjuliosilva72@gmail.com
}

\section{RESUMO}

O presente trabalho objetiva identificar a evolução das publicações sobre logística reversa nos periódicos nacionais qualificados como A1, A2, B1, B2, B3, B4 e B5 pela Coordenação de Aperfeiçoamento de Pessoal de Nível Superior (CAPES) no ano de 2012. Trata-se de um estudo bibliométrico com abordagem predominantemente quantitativa que teve como critério de seleção do extrato estabelecido no objetivo, os periódicos nacionais com publicações em meio eletrônico. De um universo de 872 periódicos nacionais de diversas áreas de estudo, 452 contém publicações, em meio eletrônico, com artigos científicos datados na década que abrange os anos de 2003 a 2012. Na busca, foram localizados 61 artigos em 36 periódicos diferentes. Dentre os resultados observa-se que as regiões sudeste e sul são as que concentram um maior número de publicação sobre o tema pesquisado, com $87 \%$ do total das publicações. Evidencia-se que 14 periódicos são responsáveis pela publicação de $64 \%$ dos artigos e que $29 \%$ das publicações se encontram em periódicos classificados como B3. No total de 170 autores identificados, 10 tiveram participação na produção de $46 \%$ dos artigos e 90\% dos autores estão vinculados a alguma Instituição de Ensino Superior, concentrados principalmente na região sudeste do país. Observa-se uma forte correlação do tema com as questões ambientais, onde 5 das 10 palavras-chave que mais aparecem estão relacionadas à responsabilidade ambiental. Quanto à evolução do tema, observa-se que o número de autores e volume de artigos publicados tem evoluído, desde 2003, onde o ápice foi o ano de 2012, com 34 autores, e 11

\footnotetext{
1 Artigo recebido em: 22/09/2013. Revisado por pares em: 10/03/2014. Segunda versão enviada em 09/09/2014. Recomendado para publicação em: 27/10/2014 por Orleans Silva Martins (Editor Geral). Publicado em: 28/11/2014. Organização responsável pelo periódico: UFPB.

2 Endereço: Rua Antônio Veiga, 140, Victor Konder, FURB - Campus 1, Sala D-202, CEP 89012-900, Blumenau/SC. DOI: http://dx.doi.org/10.18405/recfin20140304
} 
publicações. Já a relação entre teoria e prática esteve presente em $60,5 \%$ dos artigos, com destaque para o setor industrial, com 19 aplicações, e o ramo de eletroeletrônicos, com 8 aplicações. O estudo contribui ao evidenciar, no contexto nacional, autores que servem de referência para a área, periódicos que publicam sobre o tema, temáticas pouco abordadas e o indicativo de que a área continua em expansão.

Palavras-chave: Logística Reversa. Bibliometria. Meio Ambiente. Sustentabilidade. Gestão Ambiental.

\section{ABSTRACT}

This paper aims to identify the evolution of publications on reverse logistics in national professional journals such as A1, A2, B1, B2, B3, B4 and B5 of the Coordination for Improvement of Higher Education in 2012 - Qualis/CAPES 2012. It is a bibliometric study with predominantly quantitative approach was to extract selection criteria established in the objective, national periodicals with publications in electronic media. From a universe of 872 national journals in various fields of study contains 452 publications, electronic media, with papers dated in the decade spanning the years 2003 to 2012 In the search, 61 articles were located in 36 different journals. Among the results, it is observed that the southeastern and southern regions are those that concentrate a greater number of publications on the topic researched, with $87 \%$ of total publications. It is evident that 14 periodicals are responsible for the publication of articles and $64 \%$ of that $29 \%$ of the publications are in journals classified as B3. A total of 170 authors identified, 10 have participated in the production of $46 \%$ of articles and $90.0 \%$ of the authors are linked to any higher education institution, concentrated mainly in the southeastern region of the country. We observe a strong correlation with the theme of environmental issues, where five of the 10 keywords that appear most are related to environmental responsibility. As the evolution of the topic, it is observed that the number of authors and volume of articles published on the topic has increased since 2003, where the summit was the year 2012 with 34 writers and 11 publications. Yet the theory and practice relation was present in $60.5 \%$ of the articles, especially the industrial sector with 19 applications and the branch of electronics applications with eight. The study contributes to the evidence, in the national context, who authors serve as reference for the area, journals that publish on the topic, and the topics addressed some indication that the area continues to expand.

Keywords: Reverse Logistics. Bibliometrics. Environment. Sustainability. Environmental Management.

\section{INTRODUÇÃO}

Com o surgimento cada vez mais acelerado de novas tecnologias, diversos produtos se tornam rapidamente obsoletos e geram toneladas de lixo, por conseguinte, contribuem para muitos dos problemas ambientais existentes na atualidade. De acordo com o Panorama Ambiental Global 5 (GEO 5) do Programa das Nações Unidas para o Meio Ambiente, em 2007 somente os países da Organização de Cooperação Econômica e Desenvolvimento (OCDE) produziram cerca de $650 \mathrm{mi}-$ lhões de toneladas de resíduos municipais, crescendo em torno de 0,5 a 0,7 por cento ao ano, onde, deste total, de $5 \%$ a $15 \%$ são resíduos eletrônicos (GEO 5, 2012). Por causa dessa crescente escala de descartabilidade dos produtos e consequente aumento na produção de lixo, a preocupação com a recuperação, reciclagem ou correto descarte desses materiais vem aumentando gradativamente ao longo dos anos.

Para Lacerda (2002) existe uma clara tendência de que a legislação ambiental caminhe no sentido de tornar as empresas cada vez mais responsáveis pelo ciclo de vida total do produto. Esse pensamento tornou-se realidade na Lei no 12.305/2010, que trata da Gestão dos Resíduos Sólidos. Em seu Art. 3ㅜ, inciso IV, a referida Lei descreve que o ciclo de vida do produto compreende a sé- 
rie de etapas que envolvem o desenvolvimento do produto, a obtenção de matérias-primas e insumos, o processo produtivo, o consumo e a disposição final (BRASIL, 2010), demonstrando que a responsabilidade das organizações sobre os produtos fabricados não finaliza na entrega ao consumidor e sim na disposição final ou no reaproveitamento do produto.

Além das exigências legais que devem ser cumpridas, organizações que descartam incorretamente seus resíduos ou produtos geram uma imagem ruim perante a sociedade. Com isso, diversos produtos ou resíduos que anteriormente eram descartados, hoje retornam para as empresas através do fluxo logístico reverso. No entanto, de acordo com Leite (2009), esses retornos não representam somente custos, uma vez que, se trabalhados de forma correta, podem representar economia de recursos, gerando ganhos financeiros.

Mesmo com o crescimento da relevância da logística reversa em relação a questões de sustentabilidade ecológica, há pouca informação sobre o atual estado da arte das pesquisas acadêmicas relacionadas ao tema, fato que remete o estudo a investigar a seguinte questão: quais são as características bibliométricas das pesquisas que tratam da temática de logística reversa nos periódicos brasileiros? Decorrente da questão de pesquisa, o estudo tem por objetivo identificar características bibliométricas da publicação de artigos científicos em periódicos nacionais nos últimos 10 anos (2003 a 2012) que tratam do tema de logística reversa.

O estudo justifica-se pela possibilidade de evidenciar como o meio acadêmico está se posicionando em relação ao tema e verificar se o mesmo vem adquirindo destaque nos debates acadêmicos no decorrer dos anos. Busca-se, igualmente, expandir estudos anteriores que investigaram a produção científica relacionada à logística reversa socializada em eventos científicos (SANTOS et al., 2011; TENÓRIO; SILVA; DACORSO, 2014), indexadas em bases de dados (VAZ; MALDONADO; SELIG, 2013) ou baseadas em fontes mistas como no estudo de Silva Filho et al. (2009) e Pokharel e Mutha (2009). Além da introdução, este artigo possui embasamento teórico sobre a logística reversa e a pesquisa bibliométrica, seguido pela metodologia de pesquisa utilizada, pelos dados coletados no estudo bibliométrico e sua análise e, por fim, apresentam-se as considerações finais.

\section{LOGÍSTICA REVERSA}

Para o Reverse Logistics Executive Council (RLEC) logística reversa é o processo de recapturar valor ou dar destinação apropriada a produtos e materiais por meio do controle da eficiência e custo do fluxo de matérias-primas, estoques em processo e produtos acabados e a informações correspondentes do ponto de consumo ao ponto de origem (JAYARAMAN; PATTERSON; ROLLAND, 2003; RLEC, 2004). Leite (2009) complementa que logística reversa, em seu sentido mais amplo, compreende todas as operações relacionadas com a reutilização de materiais com a finalidade de agregar valor econômico, ecológico, legal e de localização.

Etapas como coleta, inspeção e separação para reciclagem ou remanufatura para posterior revenda são algumas das atividades da logística reversa. Outros retornos como devolução de clientes, retornos de embalagens, retorno de produtos ou materiais para atender a legislação, além do fluxo de informação envolvido nesse processo integram as atividades de logística reversa (LAVEZ; SOUZA; LEITE, 2011). Assim, observa-se que, na logística reversa, a responsabilidade das empresas não finaliza na entrega do produto ao cliente, pelo contrário, as empresas tornam-se responsáveis pelo retorno dos produtos aos seus estabelecimentos após o seu consumo, seja para reciclagem ou descarte, abrangendo o ciclo de vida total do produto (SOUZA; FONSECA, 2008).

Entendem-se que a logística reversa inicia no momento em que o produto é entregue ao cliente e neste momento a empresa precisa estar preparada para o que Penkar (2005) chama de os 4 R's da logística reversa, apresentados no Quadro 1. 


\begin{tabular}{|c|l|}
\hline $\begin{array}{c}\text { Fase do processo de } \\
\text { logística reversa }\end{array}$ & \multicolumn{1}{c|}{ Quadro 1 - Os 4 R's da Logística Reversa. } \\
\hline Recuperação do processo & $\begin{array}{l}\text { É o retorno do produto a empresa. Permite o controle dos estoques, as saídas e a confiabilidade } \\
\text { do produto, testa as peças defeituosas e devolve um produto melhor ao mercado. }\end{array}$ \\
\hline Reconciliação & $\begin{array}{l}\text { É a análise dos produtos que retornam a empresa para verificar em a qual canal será destinado, } \\
\text { como reparação, reposição, reconstrução ou retorno ao mercado. }\end{array}$ \\
\hline Reparação & É o tempo de espera do cliente para o produto ser trocado. \\
\hline Reciclagem & $\begin{array}{l}\text { É o retorno de produtos que seriam descartados ao ciclo de produção, reduzindo custos e abrin- } \\
\text { do novas oportunidades de negócios. }\end{array}$ \\
\hline
\end{tabular}

Fonte: Adaptado de Penkar (2005).

Com base nessas definições, novas oportunidades de negócios se abrem para as empresas que utilizam a logística reversa. Leite (2011) comenta que a logística reversa não é mais um assunto desconhecido como era anos atrás, ela adquiriu importância no mundo empresarial. Quando executada de forma eficiente, deixa de ser um problema para tornar-se um ganho sob diferentes aspectos, onde o retorno obtido através da melhoria do processo de logística reversa justifica os investimentos realizados (LACERDA, 2002).

\subsection{Canais De Pós-Venda e Pós-Consumo}

Leite (2009) identifica que o retorno dos bens ocorre por meio de canais de distribuição reversos, onde os mesmos agregam valores econômicos, ecológicos, legais, logísticos, de imagem corporativa, entre outros. $\mathrm{O}$ autor divide esses canais em de pós-venda e de pós-consumo, sendo o segundo ainda divido em canais de reuso e reciclagem. As características de cada canal estão especificadas no Quadro 2.

Quadro 2 - Os canais de pós-venda e pós-consumo.

\begin{tabular}{|c|c|c|}
\hline \multicolumn{2}{|c|}{ Canal } & Características \\
\hline \multicolumn{2}{|c|}{ Pós-venda } & $\begin{array}{l}\text { - Produto com pouco ou nenhum uso; } \\
\text { - Retornos relacionados à qualidade geral ou a processos comerciais; } \\
\text { - Motivos de retorno: término de validade, estoques excessivos em determinando canal de } \\
\text { distribuição, garantias comerciais, retorno de venda consignada, entre outros; } \\
\text { - Após o retorno são encaminhados a mercados secundários, reformas, desmanche, recicla- } \\
\text { gem ou destinação final. }\end{array}$ \\
\hline \multirow[b]{2}{*}{$\begin{array}{c}\text { Pós- } \\
\text { consumo }\end{array}$} & De reuso & $\begin{array}{l}\text { - Final de uso pelo seu primeiro possuidor; } \\
\text { - O produto ou seu componente ainda apresentam condições de uso em sua função original, } \\
\text { sendo comercializados pelos mercados de segunda mão. Ex.: mercado de veículos usados. }\end{array}$ \\
\hline & $\begin{array}{c}\text { De } \\
\text { reciclagem }\end{array}$ & $\begin{array}{l}\text { - Efetivo fim de vida útil do produto; } \\
\text { - Encaminhado ao desmanche e a reciclagem onde o produto passa por um processo de } \\
\text { desmontagem e seus componentes em condições de uso ou de remanufatura são enviados a } \\
\text { mercados de peças usadas e os aproveitáveis são enviados a reciclagem, onde são reutiliza- } \\
\text { dos na fabricação de novos produtos; } \\
\text { - Destinação final se não reaproveitável. }\end{array}$ \\
\hline
\end{tabular}

Fonte: Adaptado de Leite (2009). 


\section{BIBLIOMETRIA}

Conforme Björneborn e Ingwersen (2004) e Guedes e Borschiver (2005) a bibliometria é formada por um conjunto de leis e princípios empíricos que utilizam métodos matemáticos e estatísticos para estabelecer diretrizes de busca e classificação na pesquisa científica sobre Ciência da Informação.

Surgida na primeira metade do século XX, a bibliometria possui três leis que se destacam. Lei de Lotka, que está relacionada à produtividade dos autores por meio da identificação da frequência de publicações. Lei de Zipf, relacionada à frequência de aparecimento de palavras, medindo e gerando uma lista de termos de alta e baixa frequência. E a Lei de Bradford, que se relaciona à dispersão da produção, permitindo estabelecer núcleo e áreas de dispersão (VANTI, 2002).

Como exemplo de aplicabilidade, a Lei de Lotka, ao verificar a produtividade, auxilia na identificação dos centros de pesquisa mais desenvolvidos sobre determinado assunto e na verificação da solidez de uma determinada área científica. A Lei de Zipf (cuja $2^{\underline{a}}$ parte foi modificada por Booth) auxilia na identificação da frequência de aparecimento de determinadas palavras, onde o ponto de transição das palavras de alta frequência para as de baixa frequência representa as palavras de alto conteúdo semântico, o que auxilia na indexação dos textos. A Lei de Bradford, ao verificar núcleo ou dispersão, auxilia no desenvolvimento de políticas de aquisição e descarte de periódicos sobre determinados assuntos (POTTER, 1981; GUEDES; BORSCHIVER, 2005).

\section{METODOLOGIA}

Este estudo constituiu um levantamento bibliométrico descritivo da produção científica sobre o tema logística reversa presente nas revistas científicas publicadas na década compreendida entre 2003 e 2012. O universo de pesquisa considerado abrangeu 872 periódicos classificados como A1, A2, B1, B2, B3, B4 e B5 no ano de 2012, conforme o Qualis/CAPES (CAPES, 2012). Essa classificação é constituída por periódicos nacionais e internacionais, voltados aos mais diversificados ramos do conhecimento, como Administração, Medicina, Engenharia, Economia etc. A pesquisa procedeu-se por meio de acesso a periódicos que tivessem publicações online em língua portuguesa, onde, foram localizados 452 periódicos.

A opção de somente publicações nacionais em língua portuguesa justifica-se pela intenção do autor de verificar a evolução do tema no Brasil. A escolha de periódicos justifica-se pelo fato dos fóruns acadêmicos, a exemplo de congressos e simpósios, constituírem-se em uma publicação provisória que, após debate entre os pares, torna-se ou deveria tornar-se definitiva por meio da publicação em revistas acadêmicas. Embora a logística reversa seja originalmente uma área da Administração, ela pode ser aplicada à praticamente todas as áreas de estudo, por isso, considerou-se válido procurar o tema logística reversa em todos os periódicos nacionais que possuem suas obras publicadas em meio eletrônico.

Desta forma, foram selecionados 452 periódicos nacionais que apresentavam publicações online, onde dos quais 36 periódicos apresentaram 61 artigos que continham o termo chave logística reversa no decorrer do texto. A busca pelos artigos foi realizada através da localização do site do periódico, pela procura do tema chave e verificação do conteúdo do artigo.

Os parâmetros da pesquisa foram estabelecidos a partir da busca, leitura e análise de cada artigo, com o objetivo de evidenciar a aplicação da temática no mundo acadêmico. Portanto, a análise dos artigos selecionados constitui-se em uma análise de conteúdo, onde é possível demonstrar a evolução e o atual nível de pesquisa sobre o tema logística reversa. 


\section{DISCUSSÃO E ANÁLISE OS DADOS}

O termo "logística reversa" foi encontrado em 61 artigos, que compõem a amostra dessa pesquisa. Em 43 artigos o termo aparece no título, em 56 artigos o termo aparece nas palavras chave e em dois artigos aparece somente no corpo do texto. Ao todo foram localizadas 230 palavras chave, demonstradas na Tabela 1 em ordem decrescente de quantidade de ocorrências.

Tabela 1 - Palavras-chave.

\begin{tabular}{c|l|c|c}
\hline \multirow{2}{*}{ Item } & \multicolumn{2}{c|}{ Palavra } & \multicolumn{2}{c}{ Quantidade } \\
\cline { 3 - 4 } & & $\mathbf{N}$ & $\mathbf{\%}$ \\
\hline 01 & Logística reversa & 56 & 24,3 \\
02 & Reciclagem & 10 & 4,3 \\
03 & Gestão ambiental & 8 & 3,5 \\
04 & Sustentabilidade & 7 & 3,0 \\
05 & Logística & 6 & 2,6 \\
06 & Cadeia de suprimentos & 4 & 1,7 \\
07 & Meio ambiente & 4 & 1,7 \\
08 & Cadeia reversa & 3 & 1,3 \\
09 & Fluxo reverso & 3 & 1,3 \\
10 & Impacto ambiental & 3 & 1,3 \\
11 & Responsabilidade social & 123 & 1,3 \\
12 & Outras palavras chave & $\mathbf{2 3 0}$ & $\mathbf{1 0 0 , 0}$ \\
\hline
\end{tabular}

Fonte: Dados da pesquisa.

Conforme demonstra a Tabela 1, por ser o objeto desta pesquisa e conforme comentado anteriormente o termo logística reversa é o mais citado, com 56 termos ou 24,3\% do total das palavras-chave encontradas. Posteriormente, aparece o termo reciclagem, com 10 citações (4,3\%), seguido pelos termos gestão ambiental com 8 citações (3,5\%), sustentabilidade com 7 citações (3,0\%), logística com 6 citações (2,6\%), cadeia de suprimentos e meio ambiente com 4 citações cada (1,7\% cada) e cadeia reversa, fluxo reverso, impacto ambiental e responsabilidade social com 3 citações cada termo (1,3\% cada). A identificação das palavras-chave de maior frequência nos estudos relacionados à logística reversa pode contribuir para ampliar o conjunto de palavras de busca (tesauros) no processo de pesquisa sobre o tema.

Os artigos localizados estão publicados em 36 periódicos, o que representa uma média de 1,7 artigos por periódico. Para demonstrar os periódicos que continham artigos científicos que tratavam sobre a logística reversa foi elaborada a Tabela 2. Os periódicos estão organizados por ordem decrescente pela classificação Qualis/CAPES em 2012 e em sequência por ordem alfabética, demonstrando também o ano da primeira publicação da revista.

Observa-se que não houve publicação sobre o tema em periódicos classificados como A1. Dos 36 periódicos que compõem a base da pesquisa, 21 (58,3\%) possuem 10 anos ou menos de publicação, ou seja, pode-se considerá-los periódicos "jovens", o que de certa forma, sinaliza o crescimento do número de revistas científicas no Brasil nos últimos anos. 
Tabela 2 - Origem dos artigos da amostra.

\begin{tabular}{|c|c|c|c|}
\hline Item & Periódico & Qualis & Ano da 1a Edição \\
\hline 01 & BBR - Brazilian Business Review (Edição em português) & A2 & 2004 \\
\hline 02 & G\&P - Gestão \& Produção (UFSCAR) & A2 & 1994 \\
\hline 03 & RAC - Revista de Administração Contemporânea & A2 & 1997 \\
\hline 04 & RAE - Revista de Administração de Empresas & $\mathrm{A} 2$ & 1961 \\
\hline 05 & PRODUÇÃO - Produção (São Paulo) & B1 & 1991 \\
\hline 06 & RAI - Revista de Administração e Inovação & B1 & 2004 \\
\hline 07 & RESR - Revista de Economia e Sociologia Rural & B1 & $S / I^{*}$ \\
\hline 08 & G\&P - Gestão \& Planejamento (Salvador) & B2 & 1999 \\
\hline 09 & ORA - Organizações Rurais e Agroindustriais (UFLA) & B2 & 1999 \\
\hline 10 & P\&DEP - Pesquisa \& Desenvolvimento Engenharia de Produção & B2 & 2003 \\
\hline 11 & PRETEXTO - Pretexto (Belo Horizonte) & B2 & 2000 \\
\hline 12 & REBRAE - Revista Brasileira de Estratégia & B2 & 2008 \\
\hline 13 & RGSA - Revista de Gestão Social e Ambiental & B2 & 2007 \\
\hline 14 & RP - Revista Produção Online & B2 & 2001 \\
\hline 15 & DMA - Desenvolvimento e Meio Ambiente (UFPR) & B3 & 2000 \\
\hline 16 & GESTÃO - Gestão.Org & B3 & 2003 \\
\hline 17 & RA - Revista Alcance & B3 & 1997 \\
\hline 18 & RAU - Revista de administração da Unimep & B3 & 2003 \\
\hline 19 & REAd - Revista Eletrônica de Administração (Porto Alegre) & B3 & 1995 \\
\hline 20 & RECADM - Revista Eletrônica de Ciência Administrativa & B3 & 2002 \\
\hline 21 & RGI - Revista Gestão Industrial & B3 & 2005 \\
\hline 22 & RMPE - Revista da Micro e Pequena Empresa (FACCAMP) & B3 & 2007 \\
\hline 23 & RPCAd - Revista Pensamento Contemporâneo em Administração (UFF) & B3 & 2007 \\
\hline 24 & RU - Revista da Unifebe & B3 & $S / I^{*}$ \\
\hline 25 & ABC - ABCustos Associação Brasileira de Custos & B4 & 2006 \\
\hline 26 & GEPROS - Gestão da Produção, Operações e Sistemas & $\mathrm{B} 4$ & 2005 \\
\hline 27 & INGEPRO - Revista Ingepro: Inovação, Gestão e Produção & B4 & 2009 \\
\hline 28 & POD - Pesquisa Operacional para o Desenvolvimento & B4 & 2009 \\
\hline 29 & RACE - Revista de Administração, Contabilidade e Economia & B4 & $\mathrm{S} / \mathrm{I}^{*}$ \\
\hline 30 & RCAd - Revista Ciências Administrativas (UNIFOR) & B4 & $\mathrm{S} / \mathrm{I}^{*}$ \\
\hline 31 & RQ - Revista Qualitas & B4 & 2002 \\
\hline 32 & CENU - Cadernos da Escola de Negócios da Ubrasil & B5 & 2003 \\
\hline 33 & GS - Gaia Scientia (UFPB) & B5 & 2007 \\
\hline 34 & JP - Jovens Pesquisadores & B5 & 2004 \\
\hline 35 & RCA - Revista Científica da AJES & B5 & 2010 \\
\hline 36 & REA - Revista Eletrônica de Administração (Franca) & B5 & 2002 \\
\hline
\end{tabular}

Fonte: Dados da pesquisa. Nota: ${ }^{*}$ S/I - Sem informação: não foi possível encontrar ou determinar corretamente.

Para identificar quais os periódicos que mais publicam artigos sobre o tema logística reversa foi criada a Tabela 3, ordenada em ordem decrescente de quantidade, posteriormente por ordem alfabética, demonstrando a classificação Qualis/CAPES e o agrupando em uma única linha dos periódicos que possuem uma publicação. A análise da Tabela 3 demonstra que nos periódicos analisados foram encontrados 61 artigos que comentam sobre a logística reversa e que 14 dos 36 periódicos encontrados são responsáveis por mais da metade das publicações (63,9\%), em que a Revista G\&P, com 5 artigos (8,2\%), é a que possui o maior número de publicação, precedida pelas revistas: PRODUÇÃO e RGSA, com 4 artigos cada periódico (6,6\% cada), GEPROS, RECADM, RPCAD e RP, com 3 artigos cada periódico (4,9\% cada), e GESTÃO, JP, PRETEXTO, ERA, REAd, RMPE e RGI, com 2 artigos cada periódico (3,3\% cada). No estudo de Silva Filho et al. (2009), as Revistas G\&P e PRODUÇÃO também foram identificadas como as mais produtivas, sinalizando que elas têm mantido interesse constante pelo tema. 
Tabela 3 - Publicações por periódico.

\begin{tabular}{|c|c|c|c|c|}
\hline \multirow{2}{*}{ Item } & \multirow{2}{*}{ Periódico } & \multirow{2}{*}{ Qualis } & \multicolumn{2}{|c|}{ Quant. Artigos } \\
\hline & & & $\mathbf{N}$ & $\%$ \\
\hline 01 & G\&P - Gestão \& Produção (UFSCAR) & A2 & 5 & 8,2 \\
\hline 02 & PRODUÇÃO - Produção (São Paulo) & B1 & 4 & 6,6 \\
\hline 03 & RGSA - Revista de Gestão Social e Ambiental & B2 & 4 & 6,6 \\
\hline 04 & GEPROS - Gestão da Produção, Operações e Sistemas & B4 & 3 & 4,9 \\
\hline 05 & RECADM - Revista Eletrônica de Ciência Administrativa & B3 & 3 & 4,9 \\
\hline 06 & RP - Revista Produção Online & B3 & 3 & 4,9 \\
\hline 07 & RPCAd - Revista Pensamento Contemporâneo em Administração (UFF) & B2 & 3 & 4,9 \\
\hline 08 & GESTÃO - Gestão.Org & B3 & 2 & 3,3 \\
\hline 09 & JP - Jovens Pesquisadores & B5 & 2 & 3,3 \\
\hline 10 & PRETEXTO - Pretexto (Belo Horizonte) & B2 & 2 & 3,3 \\
\hline 11 & RAE - Revista de Administração de Empresas & A2 & 2 & 3,3 \\
\hline 12 & REAd - Revista Eletrônica de Administração (Porto Alegre) & B3 & 2 & 3,3 \\
\hline 13 & RGI - Revista Gestão Industrial & B3 & 2 & 3,3 \\
\hline 14 & RMPE - Revista da Micro e Pequena Empresa (FACCAMP) & B3 & 2 & 3,3 \\
\hline 15 & Outros periódicos apresentados da Tabela 1 (22 periódicos) & & 1 & 35,9 \\
\hline \multicolumn{3}{|c|}{ Total } & 61 & 100,0 \\
\hline
\end{tabular}

Fonte: Dados da pesquisa.

Com o intuito de demonstrar em qual classificação Qualis/CAPES encontram-se os artigos que compõem a amostra da pesquisa, elaborou-se a Tabela 4. Ela demonstra que dos 61 artigos encontrados, 9 foram em periódicos com qualificação A2, 6 em qualificação B1, 13 em qualificação B2, 18 em qualificação B3, 9 em qualificação B4 e 6 em qualificação B5, conforme classificação.

Tabela 4 - Publicações por classificação no Qualis/CAPES.

\begin{tabular}{c|c|c}
\hline \multirow{2}{*}{ Qualificação } & \multicolumn{2}{|c}{ Quantidade } \\
\cline { 2 - 3 } & $\mathbf{N}$ & $\mathbf{\%}$ \\
\hline A2 & 9 & 14,8 \\
B1 & 6 & 9,8 \\
B2 & 13 & 21,3 \\
B3 & 18 & 29,5 \\
B4 & 9 & 14,8 \\
B5 & 6 & 9,8 \\
\hline Total & $\mathbf{6 1}$ & $\mathbf{1 0 0 , 0}$ \\
\hline
\end{tabular}

Fonte: Dados da pesquisa.

Os periódicos que compõem a amostra da pesquisa estão localizados geograficamente por todo o Brasil. A Figura 1 demonstra em quais unidades da federação há maior publicação de artigos científicos com o termo "logística reversa".

Ao analisar a Figura 1, percebe-se que as publicações por periódico com o termo "logística reversa" concentram-se nas regiões Sudeste e Sul do Brasil, com destaque para o estado de São Paulo, com 26 periódicos, e o estado do Paraná, com 9 periódicos. Ao todo, os periódicos que apresentam artigos com o termo "logística reversa" ficaram distribuídos em: 35 (57,5\%) na região Sudeste, 19 (31,1\%) na região Sul, 6 (9,8\%) na região Nordeste e 1 (1,6\%) na região Centro-Oeste. A região Norte não apresentou periódicos que publiquem artigos com o termo logística reversa que estejam na classificação Qualis/CAPES. 
Figura 1 - Periódicos por Unidade da Federação.

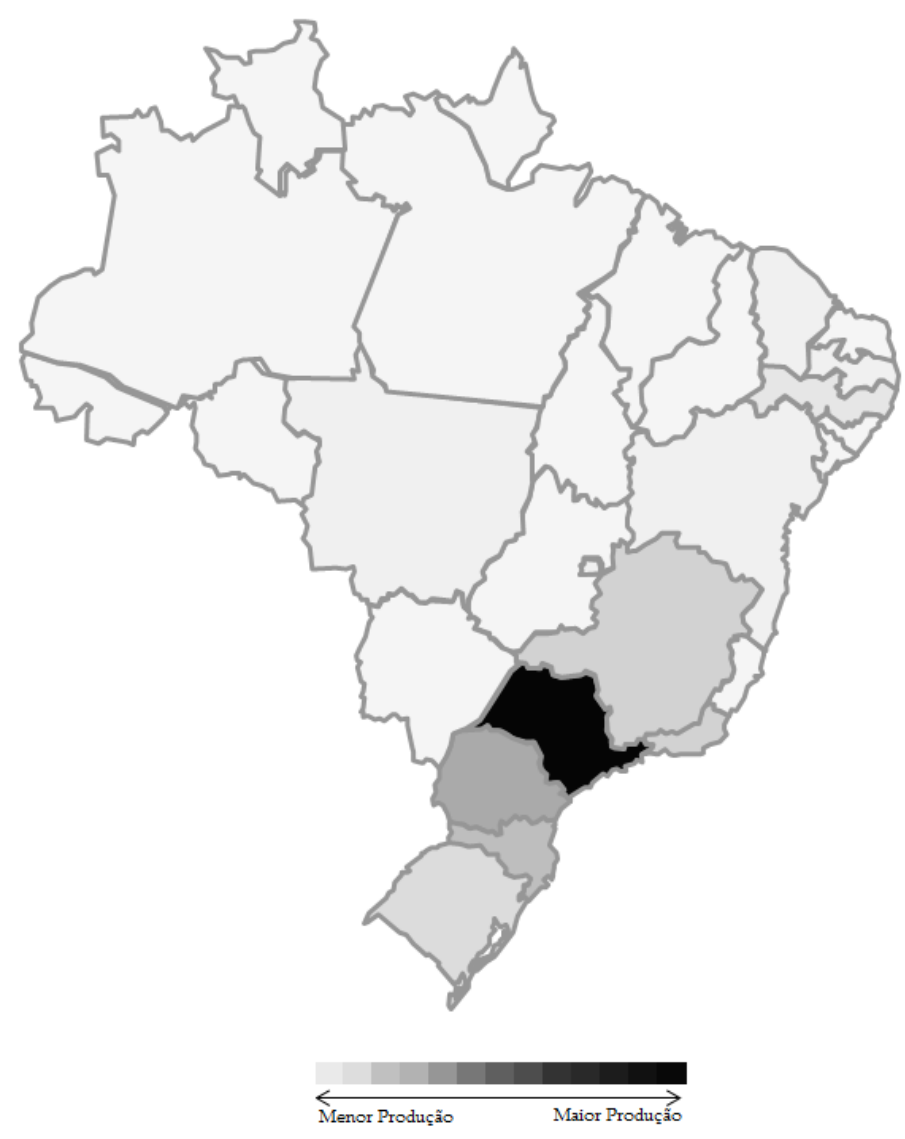

Fonte: Dados da pesquisa.

A evolução das publicações sobre a logística reversa ao longo do período analisado pode ser vista na Figura 2.

Figura 2 - Evolução das publicações sobre logística reversa.

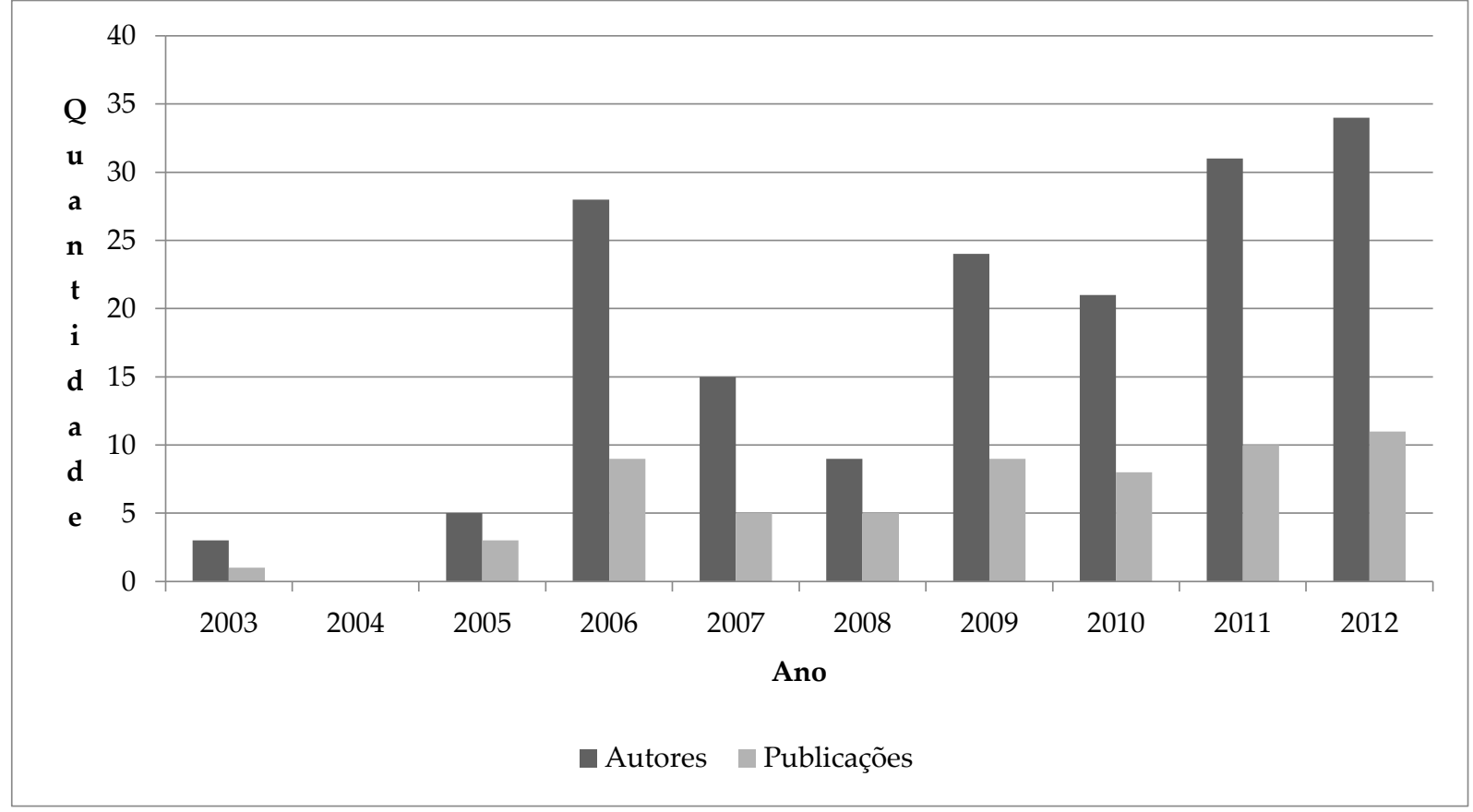

Fonte: Dados da pesquisa. 
Referente à autoria, 170 autores (152 diferentes) foram responsáveis pelos 61 artigos da amostra desta pesquisa, sendo que 27 artigos (44,3\%) foram elaborados por três autores, 19 artigos (31,1\%) por duplas de autores, 5 artigos por apenas um autor, 5 artigos por quatro autores, 4 artigos por cinco autores e 1 artigo por seis autores.

A distribuição dos autores dos artigos por periódico é evidenciada na Tabela 5, apresentada em ordem decrescente de quantidade de autores de artigos por periódico e agrupando em uma linha os periódicos com menos de quatro autores dos artigos da amostra da pesquisa.

Tabela 5 - Autores de artigos por periódico.

\begin{tabular}{l|l|c|c}
\hline \multirow{2}{*}{ Item } & \multicolumn{1}{c|}{ Periódico } & \multicolumn{2}{c}{ Quantidade Autores } \\
\cline { 3 - 4 } & & $\mathbf{N}$ & $\mathbf{1}$ \\
\hline 01 & PRODUÇÃO - Produção (São Paulo) & 11 & 7,1 \\
02 & G\&P - Gestão \& Produção (UFSCAR) & 11 & 6,5 \\
03 & RGSA - Revista de Gestão Social e Ambiental & 9 & 6,5 \\
04 & GEPROS - Gestão da Produção, Operações e Sistemas & 9 & 5,3 \\
05 & JP - Jovens Pesquisadores & 9 & 5,3 \\
06 & RPCAd - Revista Pensamento Contemporâneo em Administração (UFF) & 5,3 \\
07 & RP - Revista Produção Online & 9 & 4,7 \\
08 & RAE - Revista de Administração de Empresas & 8 & 4,7 \\
09 & RECADM - Revista Eletrônica de Ciência Administrativa & 8 & 3,5 \\
10 & GESTÃO - Gestão.Org & 6 & 3,5 \\
11 & PRETEXTO - Pretexto (Belo Horizonte) & 6 & 3,5 \\
12 & REAd. Revista Eletrônica de Administração (Porto Alegre) & 5 & 2,9 \\
13 & RAI - Revista de Administração e Inovação & 5 & 2,9 \\
14 & REBRAE - Revista Brasileira de Estratégia & 5 & 2,9 \\
15 & RMPE - Revista da Micro e Pequena Empresa (FACCAMP) & 4 & 2,4 \\
16 & POD - Pesquisa Operacional para o Desenvolvimento & 4 & 2,4 \\
17 & RCAd - Revista Ciências Administrativas (UNIFOR) & 4 & 2,4 \\
18 & RGI - Revista Gestão Industrial & 39 & 22,9 \\
\hline & Outras períodos apresentados na tabela 1 (18 periódicos) & $\mathbf{1 7 0}$ & $\mathbf{1 0 0 , 0}$ \\
\hline
\end{tabular}

Fonte: Dados da pesquisa.

Observa-se que 9 dos 36 periódicos identificados na pesquisa são responsáveis pela publicação de artigos de 86 autores (50,6\%), onde o periódico PRODUÇÃO é o responsável pela publicação de artigos de 12 autores (7,1\%); os periódicos G\&P e RGSA são responsáveis pela publicação de artigos de 11 autores, cada periódico (6,5\% cada); os periódicos GEPROS, JP, RPCAd e RP são responsáveis pela publicação de 9 autores, cada periódico (5,3\% cada); e os periódicos RAE e RECADM são responsáveis pela publicação de 8 autores, cada periódico (4,7\% cada).

A distribuição da produção por autores pode ser verificada na Tabela 6, ordenada por maior quantidade de artigos produzidos por autor e agrupando em uma única linha os autores que produziram um artigo. Identifica-se nessa Tabela que os 10 autores citados participaram da elaboração de 28 dos 61 artigos da amostra da pesquisa, representando 45,9\% das publicações e 16,5\% dos autores. Paulo Roberto Leite aparece como o maior publicador do tema da pesquisa, com 7 artigos publicados (11,5\% dos artigos e 4,1\% dos autores), sendo 2 artigos nos periódicos GESTÃO, RGSA e JP e 1 artigo no periódico RA. Na sequência aparecem Cecília Toledo Hernández, Fernando Augusto Silva Marins e Sylmara Lopes Francelino Gonçalves Dias com 3 publicações cada autor, onde individualmente representam $4,9 \%$ dos artigos e 1,8\% dos autores. 
Tabela 6 - Publicações por autor.

\begin{tabular}{c|l|c|c|l}
\hline \multirow{2}{*}{ Item } & \multirow{2}{*}{ Autores } & \multicolumn{2}{|c|}{ Quantidade } & \multicolumn{2}{c}{$\begin{array}{c}\text { Periódicos de Publicação } \\
\text { (Quantidade) }\end{array}$} \\
\cline { 3 - 4 } & & $\mathbf{N}$ & $\mathbf{\%}$ & \\
01 & Paulo Roberto Leite & 7 & 4,1 & GESTÃO (2), RGSA (2), JP (2), RA (1) \\
03 & Cecília Toledo Hernández & 3 & 1,8 & G\&P (1), P\&DEP (1), POD (1) \\
04 & Fernando Augusto Silva Marins & 3 & 1,8 & G\&P (2), P\&DEP (1) \\
05 & Sylmara Lopes Francelino Gonçalves Dias & 3 & 1,8 & PRODUÇÃO (2), G\&P (1) \\
06 & Eliane Pereira Zamith Brito & 2 & 1,2 & GESTÃO (2) \\
07 & José Carlos Lázaro da Silva Filho & 2 & 1,2 & PRETEXTO (1), RCAd (1) \\
08 & Maria Tereza Saraiva de Souza & 2 & 1,2 & RAE (1), RECADM (1) \\
09 & Mônica Cavalcanti Sá de Abreu & 2 & 1,2 & RCAd (1), PRETEXTO (1) \\
10 & Roberto Cespón Castro & 2 & 1,2 & G\&P (1), P\&DEP (1) \\
11 & Tatiana Maria Bernardo da Silva & 2 & 1,2 & RPCAd (2) \\
\hline
\end{tabular}

Fonte: Dados da pesquisa.

As publicações dos três autores supracitados somam 4 artigos no periódico G\&P, 2 artigos nos periódicos P\&DEP e PRODUÇÃO e 1 no periódico POD. Na sequência, Eliane Pereira Zamith Brito, José Carlos Lázaro da Silva Filho, Maria Tereza Saraiva de Souza, Mônica Cavalcanti Sá de Abreu, Roberto Cespón Castro e Tatiana Maria Bernardo da Silva aparecem com 2 artigos cada autor, onde individualmente representam 3,3\% das publicações e 1,2\% dos autores. A soma das publicações dos seis autores representam 2 publicações no periódico GESTÃO, PRETEXTO, RCAd e RPCAd e 1 publicação nos periódicos RAE, RECADM, G\&P, P\&DEP.

Comparando-se a produtividade dos autores identificada no estudo com as descritas por Silva Filho et al. (2009), somente os pesquisadores Paulo Roberto Leite e Sylmara Lopes Francelino Dias mantiveram posição de destaque em ambos os estudos. Na sequência, evidencia-se que muitos autores representam ou são apoiados por alguma instituição de ensino. Com o intuito de demonstrar quais as instituições mais colaboraram com os autores na publicação dos 61 artigos da amostra, tem-se a Tabela 7, que apresenta em ordem decrescente de quantidade de produção, por instituição, e agrupa em uma linha as instituições que participaram em menos que 4 artigos.

Tabela 7 - Produção por instituição.

\begin{tabular}{|c|c|c|c|}
\hline \multirow{2}{*}{ Item } & \multirow{2}{*}{ Instituição } & \multicolumn{2}{|c|}{ Quantidade } \\
\hline & & $\mathbf{N}$ & $\%$ \\
\hline 01 & Universidade Presbiteriana Mackenzie & 12 & 7,1 \\
\hline 02 & Universidade Federal do Rio Grande do Sul - UFRGS & 11 & 6,5 \\
\hline 03 & Universidade Nove de Julho - UNINOVE & 9 & 5,3 \\
\hline 04 & Universidade Federal de Santa Catarina - UFSC & 8 & 4,7 \\
\hline 05 & Universidade Federal do Ceará - UFCE & 7 & 4,1 \\
\hline 06 & Universidade Federal Fluminense - UFF & 7 & 4,1 \\
\hline 07 & Escola de Administração de Empresas de São Paulo - EAESP/FGV & 5 & 2,9 \\
\hline 08 & Fundação Educacional Inaciana Pe. Sabóia de Medeiros - FEI & 5 & 2,9 \\
\hline 09 & Centro Federal de Educação Tecnológica do Paraná - CEFET - PR & 4 & 2,4 \\
\hline 10 & Centro Universitário de Jales - UNIJALES & 4 & 2,4 \\
\hline 11 & Faculdade de Administração, Economia e Contabilidade de Ribeirão Preto - FEARP/USP & 4 & 2,4 \\
\hline 12 & Universidade Estadual de São Paulo - UNESP & 4 & 2,4 \\
\hline 13 & Universidade Federal da Bahia - UFBA & 4 & 2,4 \\
\hline 14 & Universidade Federal Rural de Pernambuco - UFRPE & 4 & 2,4 \\
\hline 15 & Outras Instituições (37 Instituições) & 65 & 38,0 \\
\hline 16 & Instituição não encontrada ou identificada & 17 & 10,0 \\
\hline \multicolumn{2}{|r|}{$\begin{array}{r} \\
\end{array}$} & 170 & 100,0 \\
\hline
\end{tabular}

Fonte: Dados da pesquisa. 
Identificou-se que 51 instituições participaram na elaboração dos 61 artigos que compõem a amostra, representando 153 autores (90,0\%). Não foi possível identificar ou encontrar vínculo institucional para 17 autores (10,0\%). Observa-se que 14 instituições representam 88 autores, com 51,8\% da produção dos artigos que compõem a amostra da pesquisa. Onde se destaca a Mackenzie com participação em 12 artigos, seguida pela UFRGS com participação em 11 artigos e, posteriormente, a UNINOVE com participação em 9 artigos, UFSC com participação em 8 artigos, UFCE e UFF com participação em 7 artigos cada instituição. Fundação Getúlio Vargas - EAESP/FGV e FEI com participação em 5 artigos cada e CEFET - PR, UNIJALES, FEARP/USP, UNESP, UFBA e UFRPE com participação em 4 artigos.

As instituições que representam os autores que publicaram os artigos da amostra da pesquisa estão localizadas em todo o território brasileiro. Baseando-se na localização das 51 instituições (Tabela 7) que colaboram com 153 autores, é possível identificar quais unidades da federação produziram mais artigos científicos sobre o tema "logística reversa", conforme Figura 3.

Figura 3 - Instituições por Unidade da Federação.

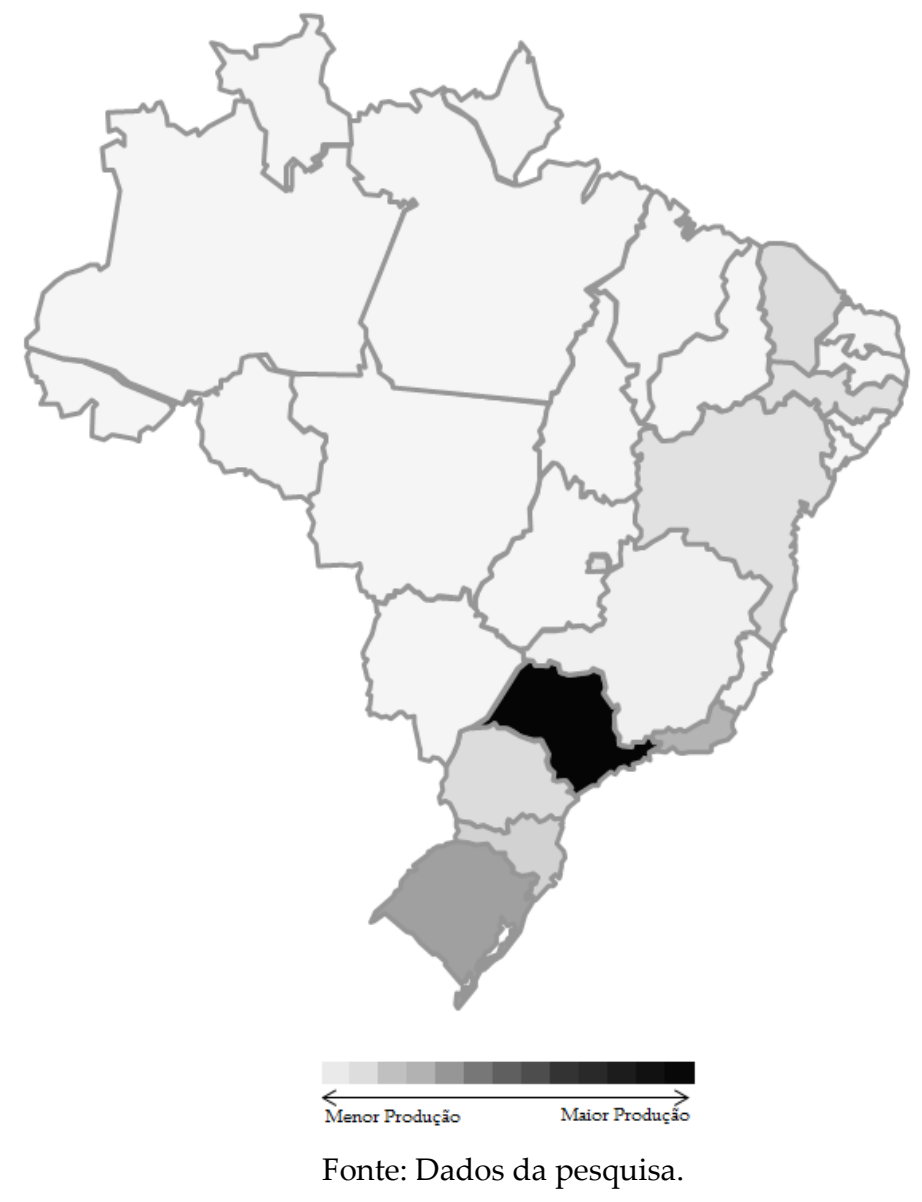

Observa-se na Figura 3 que a concentração das instituições que participaram na publicação dos artigos que compõem a amostra da pesquisa está localizada no leste do país, mais precisaram nas regiões Sudeste, Sul e Nordeste. O estado de São Paulo, seguido por Rio Grande do Sul, Rio de Janeiro e Santa Catarina, foram os que tiveram um maior número de instituição que colaboraram com os autores na publicação da amostra da pesquisa, com 64, 23, 17 e 11 autores, respectivamente, totalizando 67,6\% das autorias que compõem a amostra da pesquisa. Outras instituições localizadas no Paraná, Ceará, Bahia, Pernambuco, Distrito Federal, Paraíba e Minas Gerais também tive- 
ram contribuição nessa amostra, totalizando 36 instituições. Não houve nenhuma participação de instituições dos estados da região Norte.

Não foi possível identificar a instituição de ensino e consequentemente o estado de 17 instituições e houve também a colaboração de instituições estrangeiras nos artigos publicados por 2 autores, instituições estas localizadas em Cuba.

Nos artigos que compõem a amostra da pesquisa observou-se que muitos deles tratam da logística reversa aplicada a algum ramo de atividade específico dos diversos setores da economia. Objetivando demonstrar quais são esses ramos e a quais setores eles pertencem criou-se Tabela 8, com uma visão macro dos ramos de atividades encontrados nos artigos e seus respectivos setores. A Tabela 8 apresenta-se ordenada por ordem decrescente de quantidade por ramo.

Tabela 8 - Aplicação da logística reversa por ramo e setor.

\begin{tabular}{|c|c|c|c|c|}
\hline \multirow{2}{*}{ Item } & \multirow{2}{*}{ Ramo } & \multicolumn{2}{|c|}{ Quantidade } & \multirow{2}{*}{ Setor* } \\
\hline & & $\mathbf{N}$ & $\%$ & \\
\hline 01 & Eletro eletrônicos & 8 & 13,1 & I (6), C (1), I-S (1) \\
\hline 02 & Plásticos & 5 & 8,2 & I (3), NI (2) \\
\hline 03 & Defensivos agrícolas & 4 & 6,6 & A (2), I (1), I-C-G (1) \\
\hline 04 & Laminação de vidro & 3 & 4,9 & $\mathrm{I}(3)$ \\
\hline 05 & Bebidas & 2 & 3,3 & $\mathrm{I}(2)$ \\
\hline 06 & Borracha & 2 & 3,3 & I (1), NI (1) \\
\hline 07 & Transportes & 2 & 3,3 & $S(2)$ \\
\hline 08 & Saúde & 2 & 3,3 & $S(1), C(1)$ \\
\hline 09 & Supermercadista & 2 & 3,3 & $C(2)$ \\
\hline 10 & Alimentício & 1 & 1,6 & $\mathrm{C}(1)$ \\
\hline 11 & Artigos esportivos & 1 & 1,6 & I (1) \\
\hline 12 & Celulose e Papel & 1 & 1,6 & $\mathrm{I}(1)$ \\
\hline 13 & Energia & 1 & 1,6 & $S(1)$ \\
\hline 14 & Gráfica & 1 & 1,6 & $\mathrm{I}(1)$ \\
\hline 15 & Metalúrgico & 1 & 1,6 & $\mathrm{I}(1)$ \\
\hline 16 & Perfumaria & 1 & 1,6 & $\mathrm{C}(1)$ \\
\hline 17 & Ramo não encontrado & 24 & 39,5 & \\
\hline \multicolumn{2}{|r|}{$\begin{array}{l}\text { Total } \\
\end{array}$} & 61 & 100,0 & \\
\hline
\end{tabular}

Na Tabela 8 observa-se que 37 artigos (60,5\%) tiveram o tema "logística reversa" aplicado a um ramo específico e que o restante, 24 artigos (39,5\%), trataram o tema logística reversa de outra forma, não o relacionando com um ramo de atividade específico. Nos artigos que foi possível identificar o ramo de atividade, destaca-se o ramo de eletro eletrônicos, com 8 artigos comentando sobre o tema (13,1\%). Sendo que deles 6 foram aplicados na indústria, 1 no comércio e 1 num misto entre indústria e serviços. Posteriormente aparece o ramo de plásticos, com 5 artigos $(8,2 \%)$, sendo 3 aplicações da indústria e 3 artigos tratados de forma genérica, onde o autor deste artigo não conseguiu identificar a qual setor pertencia. Na sequência apresenta-se o ramo dos defensivos agrícolas com 4 artigos (6,6\%), sendo 2 aplicações da agricultura, 1 na indústria e 1 num misto entre indústria, comércio e governo e, depois, o ramo de laminação de vidros, com 3 artigos (4,9\%) aplicados na indústria. Os demais 17 totalizaram 7 aplicações na indústria, 5 no comércio, 4 nos serviços e 1 não identificada. Ao todo houve 19 aplicações na indústria, 6 no comércio, 4 nos serviços, 2 na agricultura, 2 mistos e 3 não identificáveis. 


\section{CONSIDERAÇÕES FINAIS}

O presente estudo tratou de uma análise dos artigos publicados em periódicos nacionais nos últimos 10 anos acerca da logística reversa, onde se efetuou a descrição das palavras-chave que mais apareceram, demonstrou-se a evolução da quantidade de publicações no decorrer dos anos e quais ramos e setores da economia os artigos se relacionaram. Referente aos periódicos demonstrou-se a origem dos artigos, quais os periódicos que mais publicaram sobre o tema, sua classificação na CAPES e localização geográfica. Quanto aos autores, evidenciou-se a quantidade de autores por artigo e, quanto aos mais produtivos, o estudo destacou o periódico que veiculou suas pesquisas, a instituição ao qual estão vinculados e sua localização geográfica.

Observa-se que na década de 2003 a 2012 as publicações sobre logística reversa foram instáveis, com períodos de alta e baixa produtividade, onde adquiriu crescimento a partir de 2009. Quanto à colaboração entre autores, foi localizado apenas um artigo que continha três autores em 2003 contra 11 artigos e 34 autores em 2012, o que sinaliza ascensão. Nota-se com isso que são recentes as pesquisas sobre o tema logística reversa, ocasionadas possivelmente pela cobrança da sociedade nos aspectos concernentes ao meio ambiente. Essa informação baseia-se nas palavraschaves encontradas nas publicações. Além da logística reversa, que foi o tema chave de busca do presente artigo, as outras 10 palavras-chave que mais aparecem nos artigos da amostra da pesquisa, cinco relacionavam-se com a responsabilidade com o meio ambiente, sendo elas: reciclagem, gestão ambiental, sustentabilidade, meio ambiente e impacto ambiental.

Nota-se que a logística reversa pode ser aplicada a qualquer ramo de qualquer setor da economia, mas os estudos tiveram uma maior aplicação da pesquisa no segmento industrial, com 19 dos 36 artigos (52,8\%), cujo ramo de negócio de maior aplicação foi o de alta tecnologia, como as de eletroeletrônicos, seguido pelo de materiais plásticos. Tal fato pode ocorrer pela legislação atribuir ao fabricante à responsabilidade do correto descarte dos produtos após o seu fim de vida útil e aos benefícios econômicos que o reaproveitamento de materiais pode causar.

Do tema pesquisado, constatou-se que 36 periódicos foram responsáveis pela publicação de 61 artigos. Quanto à distribuição destes periódicos na classificação do Qualis/CAPES, somente não foram localizados artigos sobre o tema em periódicos classificados como A1. Quanto à concentração dos artigos, 14 periódicos são responsáveis pela publicação de 63,9\% dos artigos encontrados. Os periódicos que mais se destacaram, pelo volume de publicação foram G\&P (A2), PRODUÇÃO (B1) e RGSA (B2) e constitui-se como um possível indicativo do interesse de tais periódicos a futuras pesquisas.

Em relação aos autores, foram encontrados no total 170 autores, sendo 152 autores diferentes, onde 10 destes $(6,6 \%)$ foram responsáveis pela publicação de mais de um artigo da amostra da pesquisa. O autor que mais produtivo sobre o tema é Paulo Roberto Leite, com 7 publicações. Posteriormente destacam-se Cecília Toledo Hernández e Sylmara Lopes Francelino Gonçalves Dias, com 3 publicações cada autora. Na tangente da coautoria, destaca-se que 91,8\% dos artigos da amostra da pesquisa foram produzidos por mais de um autor.

Referente às instituições de ensino que colaboram ou representam os autores, identificou-se que do total de 170 autores, 153 deles $(90,0 \%)$ tinham relação com alguma instituição de ensino. Destas instituições, as que mais se destacaram foram a Mackenzie, UFRS e UNINOVE. Baseado na localização das instituições que colaboram com os autores, foi possível determinar quais regiões do país são as que mais produzem artigos sobre logística reversa, onde São Paulo tem destaque como maior produtor com 64 dos 153 autores (41,8\%). 
A principal contribuição do presente estudo foi evidenciar as tendências de publicações sobre a temática da pesquisa, que vem apresentando um gradual crescimento desde 2009, após queda nos anos anteriores. Esse crescimento de produção vem sendo liderado por autores da área, como Paulo Roberto Leite, que também possui livros publicados pelo tema, o que o confere como um dos autores nacionais relevantes para estudos sobre logística reversa. Nota-se adicionalmente a ocorrência de uma relação da temática de logística reversa com as questões ambientais. Pela aplicação do tema em diversos ramos e setores da economia nacional percebe-se que existe uma boa relação entre teoria e prática, o que permite estudos mais aprofundados em relação à temática. Estudos que valorizem uma prática capaz de contribuir com o meio ambiente ao mesmo tempo em que traz rentabilidade para o acionista, através da otimização dos recursos existentes e atendimento a todos os stakeholders.

Por fim, os resultados da pesquisa demonstram que há uma evolução no debate acadêmico em relação à temática da pesquisa, com ênfase na prática aplicada em diversas realidades organizacionais. Com isso, através da identificação quantitativa de autores, artigos e periódicos relacionados ao termo chave, espera-se que este artigo possa contribuir com futuros trabalhos que precisem fundamentar-se com a produção e publicação nacional sobre logística reversa. Assim como fica a sugestão de estudos qualitativos aplicados a ramos de negócios diferentes dos apresentados na Tabela 8 ou então a realização de um comparativo entre trabalhos do mesmo ramo e, um levantamento das publicações internacionais, com o intuito de identificar as tendências e verificar se há harmonia entre os padrões internacionais e os nacionais.

\section{REFERÊNCIAS}

BJÖRNEBORN, L.; INGWERSEN, P. Toward a basic framework for webometrics. Journal of the American Society for Information Science and Technology, v. 55, n. 14, p. 1216-1227, 2004.

BRASIL. Lei n. 12.305, de 02 de agosto de 2.010. Institui a Política Nacional de Resíduos Sólidos; altera a lei n. 9.605, de 12 de fevereiro de 1.998; e dá outras providências. Disponível em:

http://www.planalto.gov.br/ccivil 03/ ato2007-2010/2010/lei/112305.htm. Acesso em: 19 dez. 2012.

JAYARAMAN, V.; PATTERSON, R. A.; ROLLAND, E. R. The design of reverse distribution networks: models and solution procedures. European Journal of Operational Research, v. 150, n. 1, p. 128149, 2003.

GUEDES, V. L. S.; BORSCHIVER, S. Bibliometria: uma ferramenta estatística da informação e do conhecimento, em sistemas de informação, de comunicação e de avaliação científica e tecnológica. São Paulo. 2005. Disponível em: http://www.feg.unesp.br/ fmarins/seminarios/Material\%20de\%20Leitura/Bibliometria/Artigo\%20 Bibliometria\%20\%20Ferramenta\%20estat\%EDstica\%20VaniaLSGuedes.pdf. Acesso em: 02 mar. 2013.

LACERDA, L. Logística reversa: uma visão sobre os conceitos básicos e as práticas operacionais. Revista Tecnologística, São Paulo, n. 74, p. 46-50, 2002.

LEITE, P. R. Logística reversa: meio ambiente e competitividade. 2. ed. São Paulo : Pearson, 2009. 
LEITE, P. R. Logística reversa e a regulamentação da política nacional de resíduos sólidos. Revista Tecnologística, São Paulo, 2011. Disponivel em: http://www.tecnologistica.com.br/artigos/logisticareversa-e-a-regulamentacao-da-politica-nacional-de-residuos-solidos/. Acesso em: 17 set. 2012.

LEITE, P. R.; LAVEZ, Natalie; SOUZA, Vivian Mansano de. O papel da logística reversa no reaproveitamento do "lixo eletrônico": um estudo no setor de computadores. Revista de Gestão Social e Ambiental, São Paulo, v. 5, n. 1, 2011.

NAÇÕES UNIDAS. Panorama Ambiental Global 5. 2012. Disponível em: http://www.pnuma.org.br/admin/publicacoes/texto/GEO5 RESUMO FORMULADORES POLITI CAS.pdf. Acesso em: 10 nov. 2012.

PENKAR, R. The 4 R's of reverse logistics. MHEL News, Cleveland, 12 de julho 2005. Disponível em: http://mhlnews.com/transportation-amp-distribution/4-rs-reverse-logistics. Acesso em: 25 out. 2012.

POKHAREL, S.; MUTHA, A. Perspectives in reverse logistics: a review. Resources, Conservation and Recycling, v. 53, n. 4, p. 175-182, 2009.

POTTER, W. G. Lotka's law revisited. Library Trends, v. 30, n. 1, p. 21-39, 1981.

RLEC - Reverse Logistics Executive Council, 2004. Disponível em: http://www.rlec.org/glossary.html. Acesso em: 12 set. 2012.

SANTOS, M. S.; FERNANDES, C. A.; AZEVEDO, É. S. F.; HOLANDA, L. M. C. Análise da produção científica em logística reversa nos artigos do encontro nacional de engenharia de produção. In: ENEGEP, 8, 2011. In: Anais... Resende: SEGeT, 2011.

SILVA FILHO, J. C. L.; CANTALICE, F. L. B. M.; BARBOSA JÚNIOR, C. D. S. C.; ABREU, M. C. S. Revisando o conceito de logística reversa e sua importância para o meio ambiente: análise longitudinal da produção científica entre 2003 e 2008. In: ENGEMA, 11, 2009. Anais... ENGEMA, 2009.

SOUZA, S. F.; FONSECA, S. U. L. Logística reversa: Oportunidades para redução de custos em decorrência da evolução do fator ecológico. 2008. Disponível em:

http://www.ead.fea.usp.br/semead/11semead/resultado/an_resumo.asp?cod trabalho=87. Acesso em: 15 ago. 2012.

TENÓRIO, F. A. G.; SILVA, D. E. P.; DACORSO, A. L. R. Inovação e tomada de decisão no processo de logística Reversa: uma análise bibliométrica. Revista Produção Online, v. 14, n. 2, p. 593-616, 2014.

VANTI, N. A. P. Da bibliometria à webometria: uma exploração conceitual dos mecanismos utilizados para medir o registro da informação e a difusão do conhecimento. Revista Ciência da Informação, Brasília, v. 31, n. 2, 2002. 
VAZ, C. R.; MALDONADO, M. U.; SELIG, P. M. Revisão de literatura estruturada da produção científica Sobre o conceito de logística reversa (1990-2012). In: SIMPOI, 2012. Anais... SIMPOI, 2013. 